\title{
C O N T E M P O R A R Y E P I G R A P H I C S T U D I E S
}

STUDIA EUROPAEA GNESNENSIA 16/2017

ISSN 2082-5951

DOI 10.14746/seg.2017.16.3

\author{
Silvia Orlandi \\ (Roma) \\ UN SUPPLEMENTUM AL SUPPLEMENTUM: \\ ISCRIZIONI IMPERIALI DI ROMA DAGLI ANNI '90 A OGGI
}

\begin{abstract}
In this survey article are collected and discussed most of the imperial inscriptions found in Rome after the pubblication of the last Supplement to CIL, VI by Géza Alföldy in 1996, together with a large number of possible corrections of the already published texts. The contributions are organized according the following schema: Addenda et corrigenda: small corrections, accidental gaps, methodological inconsistencies, texts to be removed. New texts and new studies: new discoveries (including new fragments of old inscriptions), both in archaeological excavations and in archives and repositories, just published (as imperial inscriptions or not) or still unpublished.
\end{abstract}

\section{Key words}

imperial inscriptions, Rome, CIL VI, Supplement, archaeological excavations, archives, epigraphic database 
Nel primo fascicolo del VI volume del CIL, curato da Bormann e Henzen nel 1876, le Inscriptiones Augustorum domusque Augustae occupano i numeri da 872 a 1230 , per un totale - tenuto conto delle epigrafi con più redazioni sullo stesso supporto - di circa 360 testi. A questi si aggiungono le diverse pagine di Additamenta (pp. 840-847) pubblicate in coda a questo stesso fascicolo, in cui si dà rapidamente conto dei nuovi studi e delle nuove scoperte, sia con una serie di addenda a pezzi già editi, sia con una tempestiva, anche se sommaria, edizione di nuovi testi e frammenti (37453822). Tutte queste nuove epigrafi troveranno poi una più compiuta edizione, insieme ad altri testi, nel primo vero e proprio supplemento a CIL, VI, curato da Hülsen nel 1902. In questo fascicolo, le iscrizioni imperiali occupano poco meno di 40 pagine (da p. 3070 a p. 3106), che comprendono, anche in questo caso, sia una serie di addenda et corrigenda, più o meno ampi (31188-31264), sia una serie di circa 270 iscrizioni pubblicate o ripubblicate ex novo (da CIL, VI 31265 a 31536). La stessa formula si ripropone poco più di 30 anni dopo, nel 1933, nell'edizione del nuovo supplemento a CIL, VI curato da Martin Bang. Anche qui a una serie di addenda (da 36880 a 36905) segue l'edizione di oltre un centinaio di nuovi testi (da 36906 a 37021$)$.

Dopo un intervallo di tempo molto più lungo di quello che aveva diviso sia l'edizione del primo fascicolo da quella del primo supplemento, sia quest'ultimo dal fascicolo di Bang, nel 1996, esce il primo volume, curato da Géza Alföldy, del nuovo Supplementum a CIL, VI, con la numerazione pars octava, fasciculus alter che lascia il posto per il fascicolo delle sacrae allora in corso di elaborazione da parte di Silvio Panciera.

Già a prima vista, la nuova era del Corpus inaugurata dalla direzione di Géza appare in tutta la sua importanza nella forma e nel contenuto di questo volume: addenda non solo bibliografici corposi e ricchi di informazioni, edizione di nuovi testi accompagnati da ampi commenti e da un notevole apparato fotografico e grafico, adozione sistematica del sistema di trascrizione internazionalmente conosciuto come sistema di Leiden, perfezionato, proprio per l'edizione del Corpus, da Silvio Panciera e Hans Krummrey. Un'autentica rivoluzione nella storia del CIL, che segna un punto di non ritorno per tutte le edizioni epigrafiche successive, che a questo modello si ispirano riconoscendone la validità. Apparentemente, quindi, un'opera destinata a rimanere un punto di riferimento e a conservare la sua attualità se non per sempre, almeno molto a lungo, come se vi fosse una corrispondenza 
tra il tempo trascorso nell'attesa dell'uscita di questo nuovo volume, 60 anni appunto, e la sua potenziale validità per il futuro.

In realtà, nei poco più di vent'anni trascorsi dall'edizione di quel fascicolo a oggi, la messe di nuovi dati emersi nell'epigrafia imperiale urbana è veramente notevole ed è quello che mi ha indotto a riflettere sul ruolo avuto da Géza in questo campo, e su quello che può avere chi, come me e, penso, molti di noi, nel solco del suo insegnamento si muove.

Nonostante il titolo un po' provocatorio del mio intervento, non ho naturalmente né l'intenzione né la possibilità di presentare in questa sede un vero e proprio „Supplementum al Supplementum”, cioè l'intero corpus dei nuovi testi scoperti o integralmente ristudiati dal 1996 a oggi. Vorrei piuttosto concentrare la mia attenzione, attraverso un cospicuo numero di esempi, sulle diverse modalità in cui le varie novità, aggiunte e correzioni sono intervenute a rendere modificabile e migliorabile l'edizione di Alföldy, per riflettere sul valore che un'impresa editoriale come il Corpus Inscriptionum Latinarum può continuare ad avere nel XXI secolo.

Considerate nel loro complesso, le novità emerse in questi anni nel campo delle epigrafi imperiali urbane, a seconda della loro genesi e della loro entità, possono essere così schematizzate:

Addenda et corrigenda:

- Piccole correzioni,

- Lacune accidentali,

- Incongruenze metodologiche,

- Testi da espungere.

Novità:

- Nuovi studi di vecchie iscrizioni,

- Nuove scoperte:

- Nuovi frammenti di vecchie iscrizioni,

- Nuovi testi:

o Editi come iscrizioni imperiali,

o Editi ma non adeguatamente valorizzati,

o Ancora inediti.

\section{ADDENDA ET CORRIGENDA}

Per quanto riguarda la voce „aggiunte e correzioni”, bisogna innanzi tutto chiarire che il fascicolo delle iscrizioni imperiali, così come quello dei 
Magistratus populi Romani uscito pochi anni più tardi, non avrebbe mai potuto essere completato in un numero così limitato di anni (considerata la mole del lavoro) se Alföldy e i suoi collaboratori non avessero potuto avvalersi dell'archivio di aggiornamento a CIL, VI creato da Silvio Panciera e tenuto costantemente aggiornato presso la Cattedra di Epigrafia Latina della Sapienza, cosa che viene esplicitamente ricordata nel frontespizio del volume, dove si legge "titulos et imagines collegit, schedasque comparavit Silvio Panciera, edidit Géza Alföldy".

Questo, però, significa anche che là dove lo schedario della Sapienza conteneva errori o imprecisioni, dovuti a sviste, dimenticanze o anche alla perdita accidentale o all'errato posizionamento di una scheda, questi errori o imprecisioni spesso si riflettono anche nell'edizione del Corpus. a volte sono stati corretti in occasione delle verifiche e dei controlli condotti da Alföldy e dai suoi collaboratori direttamente sui materiali e sulle fonti bibliografiche e d'archivio, ma non sempre ciò è stato possibile, e qualcosa è inevitabilmente sfuggito.

Ad un mancato aggiornamento dell'archivio cartaceo o, meglio, al mancato spostamento degli addenda in un'altra sezione dello schedario, ad esempio, si deve il fatto che figuri ancora tra le iscrizioni imperiali il testo pubblicato in CIL, VI 31453 e ripreso tra gli addenda et corrigenda a p. 4348, dove viene dato come irreperibile e attribuito a un imperatore incerto tra l'età di Adriano e quella di Settimio Severo, Caracalla e Geta. Il suo ricongiungimento con altri frammenti, anch'essi editi in CIL, VI, ma conservati in magazzini diversi, infatti, aveva già da tempo consentito a Mercedes Giovinazzo e Silvio Panciera di riconoscerlo come pertinente ad un'epigrafe onoraria dedicata a una vestale massima, offrendo un'edizione notevolmente migliorata del testo ${ }^{1}$, non sempre confluita nella bibliografia successiva ${ }^{2}$.

${ }^{1}$ M. Giovinazzo, S. Panciera, [in:] Iscrizioni greche e latine del Foro Romano e del Palatino (Tituli 7), Roma 1996, pp. 344-346, nr. 117, ripubblicata in S. Panciera, Epigrafi, epigrafia, epigrafisti. Scritti vari editi e inediti (1956-2005) con note complementari e indici, Roma 2006, pp. 1147-1148: ------ / [--- v(irgini) V(estali) m(aximae)] / san[ctissimae et religiosissimae] / ob ex[imi]am eius [erga se benevolentiam] / sincer[a]que ad [aeternos ignes?] / adqu[e] in divi[na altaria pietatem?] / amicitia[e] c[ausa].; cfr. AE 1996, 114 (dove figura come addendum di CIL, VI 32425). Per foto e altre informazioni vd. EDR114545. D'ora in poi, le iscrizioni saranno citate con il rinvio alla relativa scheda nella banca dati on line EDR (Epigraphic Database Roma [online]: $<$ www.edr-edr.it $>$ ), dove i lettori potranno trovare dati, bibliografia e immagini delle epigrafi considerate.

${ }^{2}$ Vd. ad esempio N. Mekacher, Die vestalischen Jungfrauen in der römischen Kaiserzeit, Wiesbaden 2006, p. 208. 
Non ad una lacuna, ma all'inserimento della scheda cartacea nella sezione dello schedario relativa agli Officiales hominum privatorum è sicuramente dovuta anche la mancata inclusione, tra i tituli imperatorum, di una lastra iscritta, parzialmente ricomposta da più frammenti, rinvenuta nell'area di S. Omobono e pertinente verosimilmente alla pubblicazione di un rescritto imperiale, forse di età costantiniana, relativo al collegio dei fabri ${ }^{3}$. Se ne fosse stato a conoscenza, Géza Alföldy non avrebbe sicuramente mancato di dedicare la dovuta attenzione a questo testo, che meriterebbe senz'altro ulteriori studi ed approfondimenti.

Un altro esempio è offerto dal caso di un'iscrizione imperiale tarda, incisa su una lastra marmorea tagliata in forma circolare per essere reimpiegata nel pavimento cosmatesco di S. Francesca Romana. Pubblicata per la prima volta in CIL, VI 31501, con un apografo fondamentale per la sua comprensione, è stata ripresa nel Supplemento con un addendum a p. 4349, firmato dallo stesso Géza. Qui si dice che la pietra è andata perduta, dando fede alle parole del parroco di S. Francesca Romana, ma, evidentemente, senza eseguire un controllo autoptico nella stessa chiesa. L'epigrafe, infatti, si conserva tuttora là dove fu vista e descritta dal Gatti e dal De Rossi nell'800, e dove Margherita Dottori l'ha recentemente fotografata, consentendoci, così, di aggiornare il nostro archivio, dove evidentemente non era stata registrata.

Lo stesso deve essere avvenuto per le varie iscrizioni imperiali date per disperse e rintracciate nel corso della sistematica ricognizione dei palazzi storici romani ad opera della Soprintendenza Archeologica di Roma, che ha portato alla pubblicazione di un volume fotografico specificamente dedicato alle collezioni epigrafiche ivi conservate ${ }^{4}$ : un'epigrafe onoraria per Caracalla e Giulia Domna è murata nella parete esterna della serra di Palazzo Barberini $^{5}$; un frammento di iscrizione imperiale di incerta interpretazione, vista per la prima volta a Palazzo Castellani, si trova ora nel Casale Santambrogio di vicolo delle Sette Chiese ${ }^{6}$; il frammento di una dedica posta

\footnotetext{
${ }^{3}$ Edito da R. Ambrosino, Riferimenti all'ordinamento associativo romano, Bullettino della Commissione Archeologica Comunale 67, 1939, pp. 85-94 = AE 1941, 68 (da cui è confluito in EDR073463). L'iscrizione è in corso di studio da parte di John Fabiano, che l'ha presentata in un paper dal titolo „Where have all the fabri tignarii gone? CIL XIV 4365 \& 4382, a reassessment of the fabri tignarii in Rome and Ostia in the early $4^{\text {th }}$ century CE", nel corso del SCS/AIA Joint Meeting tenutosi a Toronto (5-8 gennaio 2017).

${ }^{4}$ M. Bertinetti (cur.), Supplementa Italica - Imagines, Roma (CIL, VI) 5. Collezioni urbane dei palazzi storici, Roma 2016.

${ }^{5}$ M. Bertinetti (cur.), Supplementa Italica, CIL, VI $1070=$ EDR105716.

${ }^{6}$ CIL, VI 1213 = EDR112134.
} 
all'imperatore Decio e a sua moglie Etruscilla, rinvenuta nelle fondamenta del Villino Hüffer, in via Nazionale, si conserva tuttora nello stesso luogo ${ }^{7}$.

Tutt'altro che perduta, ma ancora visibile, sia pure con qualche difficoltà, sulle arcate esterne rivolte a monte del Ponte Cestio è anche la lunga iscrizione, su un'unica riga, che commemora il restauro del ponte ad opera degli imperatori Valentiniano, Valente e Graziano ${ }^{8}$. Gli autori dell' addendum a p. 4332 la danno come irreperibile probabilmente perché quella che cercavano non era un'epigrafe incisa direttamente sui blocchi del ponte, ma una lastra, simile a quella inserita a metà della spalletta in occasione dello stesso restauro9. Ma, anche in questo caso, Valentina Gorla, in occasione della sua tesi di laurea dedicata proprio alle iscrizioni tuttora visibili sui monumenti del centro di Roma ${ }^{10}$, ha potuto riconoscerla e fotografarla, recuperando, così, alla fruizione diretta un importante documento dell'attività edilizia nella Roma del IV secolo.

Allo stesso modo, Margherita Serra, nel corso delle ricerche per la sua tesi ${ }^{11}$, ha ritrovato nel pavimento di S. Clemente, dove era sempre stato e dove Henzen e De Rossi l'avevano visto, il frammento inferiore - dato per perduto a p. 4309 - di un'iscrizione parzialmente conservata ai Musei Capitolini, che menziona la nipote di Vespasiano Flavia Domitilla ${ }^{12}$. Si tratta di un testo di incerta e problematica interpretazione, che forse, grazie al ritrovamento del frammento $b$, potrà ora essere riconsiderato e meglio compreso.

Questo, infatti, è ciò che è accaduto all'iscrizione CIL, VI 40744, pubblicata nel Supplementum solo sulla base di uno scarno appunto del „Registro Trovamenti" del Comune di Roma ${ }^{13}$, e interpretata come un'iscrizione posta da un [--- Inv] ictus Aug(ustus) e datata al mese di marzo ([--- M]arti[as?]). Il ritrovamento del pezzo nel Palazzo dei Cavalieri di Rodi ha permesso

\footnotetext{
${ }^{7}$ CIL, VI $31376=$ EDR113652.

${ }^{8}$ CIL, VI $1176=$ EDR103849.

${ }^{9} \mathrm{CIL}, \mathrm{VI} 1175=$ EDR103848.

${ }^{10} \mathrm{~V}$. Gorla, Materiali per un catalogo virtuale delle iscrizioni non musealizzate di Roma. Tesi di Laurea Magistrale, Archeologia discussa nell'anno accademico 2009/2010 (relatore: S. Orlandi; correlatore: G.L. Gregori).

${ }^{11}$ M. Serra, Le iscrizioni della basilica superiore di San Clemente. Tesi di Laurea Magistrale, Archeologia discussa nell'anno accademico 2016/2017 (relatore: D. Nuzzo; correlatore: M.L. Caldelli).

${ }^{12}$ CIL, VI $948=$ EDR103964.

${ }^{13}$ Già pubblicato in precedenza da P. Virgili, Notiziario di scavi e scoperte in Roma e Suburbio 1946-1960, 2, Foro di Nerva, Bullettino della Commissione Archeologica Comunale 90, 1985, p. 321, e da C. Morselli, E. Tortorici, Curia, Forum Iulium, Forum Transitorium, Roma 1989, I, p. 118 , nt. 423 .
} 
a Laura Chioffi di proporre una nuova, più corretta lettura del testo, che è stato interpretato come una dedica a Marte posta, in occasione di una vittoria, da un imperatore, che potrebbe essere identificato con Severo Alessandro dopo il suo trionfo sui Persiani del $233^{14}$.

A importanti precisazioni nella lettura del testo e soprattutto a una nuova consapevolezza della storia post-classica dell'obelisco lateranense ha portato anche la scoperta, per merito di Roberta Marchionni, di alcuni frammenti dell'iscrizione di dedica di Costanzo II sull'obelisco stesso ${ }^{15}$, dove erano stati utilizzati nel XVI secolo per colmare alcune lacune nella pietra in occasione del recupero e del riallestimento del monumento ${ }^{16}$.

Non imputabili ad errori od omissioni, ma semplicemente ai numerosi spostamenti di materiali nell'ambito dei musei e dei magazzini delle varie soprintendenze archeologiche di Roma, sono invece i numerosi aggiornamenti che sarebbe necessario apportare ai dati relativi alle attuali collocazioni dei pezzi. Molti sono, infatti, i cambiamenti intervenuti negli ultimi anni, sia in seguito all'inaugurazione o alla riorganizzazione di diversi spazi museali come la sezione epigrafica del Museo Nazionale Romano alle Terme di Diocleziano o il nuovo allestimento della collezione epigrafica capitolina dopo lo smantellamento della vecchia Galleria Lapidaria - sia a causa della sostanziale inagibilità di alcune strutture come l'Antiquarium Comunale del Celio - solo in parte sostituito dai magazzini della Centrale Montemartini o il Museo della Civiltà Romana, dove erano state portate le casse piene di frammenti epigrafici un tempo conservate nei sotterranei del Palazzo delle Esposizioni. Ma tenere traccia di tutti questi spostamenti è impresa pressoché disperata, che cerchiamo di realizzare, almeno in parte, al momento dell'immissione delle informazioni relative al luogo di conservazione nelle schede della banca dati EDR, ma non sempre con risultati soddisfacenti e duraturi.

Altre lacune, invece, sono piuttosto da attribuire ad un cambiamento in corso d'opera nei criteri di selezione dei materiali da pubblicare nei supplementi. Nel fascicolo relativo ai Magistratus populi Romani, infatti, troviamo riedite in CIL, VI alcune iscrizioni di committenza cristiana già

\footnotetext{
${ }^{14}$ L. Chioffi, Epigrafi tra Roma e Anzio: note a margine, Rendiconti della Pontificia Accademia Romana di Archeologia 88, 2015-2016, pp. 424-430, nr. I. 1; cfr. EDR125321, dove è ora confluita la nuova lettura del testo.

${ }^{15}$ CIL, VI 1163 = EDR122871.

${ }^{16}$ R. Marchionni, La tradizione non solo manoscritta del Carmen epigraphicum patris opus munusque suum (CIL VI 1163). I segreti dell'obelisco lateranense, Rendiconti della Pontificia Accademia Romana di Archeologia 85, 2012-2013, pp. 455-472.
} 
presenti nelle ICUR o nelle ILCV, selezionate in base al fatto che menzionano senatori, cavalieri o comunque titolari di cariche di un certo rilievo, specialmente se attivi nel campo della costruzione o del restauro di opere pubbliche. Se questo criterio di selezione fosse stato usato già nel fascicolo delle imperiali, avremmo dovuto trovare, tra le iscrizioni più tarde, anche alcuni testi di notevole importanza, che invece mancano del tutto. Penso, ad esempio, all'iscrizione posta da Valentiniano III, Galla Placidia e Onoria per commemorare un restauro, eseguito a scioglimento di un voto, nella basilica di S. Croce in Gerusalemme (ILS 817 = ILCV 1775), o alla dedica votiva posta più o meno negli stessi anni a $\mathrm{S}$. Pietro in Vincoli dalla moglie di Valentiniano III, Eudossia, a nome suo e dei suoi genitori, gli imperatori Teodosio II ed Eudocia (ILS 819 = ILCV 1779). Queste mancano in CIL, VI, pur essendo del tutto simili - per contesto, forma e contenuto - alle iscrizioni relative, ad esempio, alla basilica di S. Pietro posta dal senatore Rufius Viventius Gallus o al restauro del sacello del martire Liberale ad opera del console Florus ${ }^{17}$.

Sono convinta, inoltre, che un sistematico riesame della sezione dei Fragmenta in urbe reperta vel adservata consentirebbe il recupero di diversi testi ascrivibili - per contenuto, altezza delle lettere, tecnica di scrittura - alla categoria delle iscrizioni relative ai membri della domus Augusta, come dimostrano un paio di casi particolarmente significativi.

Tra i frammenti noti solo da tradizione manoscritta, ad esempio, era stato pubblicato un grosso blocco di marmo con lettere alveolate di notevoli dimensioni ${ }^{18}$, che, ad uno studio più approfondito sull'originale oggi conservato ai Musei Vaticani, si è rivelato quel che resta, probabilmente, dell'iscrizione di dedica del Teatro di Marcello ${ }^{19}$.

Altrettanto fruttuose si sono rivelate le recentissime indagini condotte sul cosiddetto Arco di Giano, al cui attico è stato possibile attribuire un'altra iscrizione imperiale riconoscibile in alcuni frammenti conservati nella chiesa di S. Giorgio al Velabro e pubblicati tra le epigrafi frammentarie della Regio $X I$ - Circus Maximus ${ }^{20}$. Si tratta di una serie di grosse lastre marmoree,

${ }^{17}$ Pubblicate, rispettivamente, in CIL, VI 41400 = EDR093622 e CIL, VI 41434 = EDR093659.

${ }^{18}$ CIL, VI 30681, 1 = EDR073860.

${ }^{19}$ S. Orlandi, Un'iscrizione monumentale dall'area del Teatro di Marcello, [in:] G. Paci (cur.), Contributi all'epigrafia di età augustea. Actes de la XIII ${ }^{\mathrm{e}}$ Rencontre franco-italienne sur l'epigraphie di monde romain, Tivoli 2007, pp. 201-216, seguita da A. Monterroso, La scenae frons ne los teatros de Roma. Entre liturgia, formas y modelos, [in:] La scenae frons en la arquitectura teatral romana, Cartagena 2009, pp. 48-50.

${ }^{20}$ CIL, VI 30364, 4-7 = EDR162746. 
rilavorate sul retro, nel IX secolo, per ricavarne un pluteo, forse pertinente alla fase della chiesa dell'epoca di Gregorio IV (827-844) ${ }^{21}$. Le lettere monumentali incise sulla fronte, benché di incerta integrazione, fanno sicuramente riferimento ad un imperatore ${ }^{22}$ (Aug(usto) Maxi[mo]), che si è reso in qualche modo benemerito di un gesto di „liberazione” ([--- totius orbis te]rrae liber[atori--]), forse da un tiranno (azione cui si riferisconol'aggettivo superb[---], che si legge in r. 3, e l'epiteto [extinct?] ori, ricostruibile nella riga successiva), tornando a reggere le "briglie" (habenis) dello Stato. Le ipotesi di interpretazione e datazione del testo, che finora oscillavano fra l'età di Costantino e quella di Costanzo $\mathrm{II}^{23}$, hanno ora ricevuto una dirimente conferma dal rinvenimento del frammento di una base dedicata a Costantino I reimpiegata in uno dei pilastri dell' $\operatorname{arco}^{24}$, che consente di fissare al 337 il terminus post quem per la costruzione del monumento. Nell'Arco di Giano sarà dunque da riconoscere l'Arcus Divi Constatini menzionato nei Cataloghi Regionari, decretato a Costanzo II dopo la vittoria sull'usurpatore Magnenzio (353) e probabilmente inaugurato in occasione della sua visita a Roma nel $357^{25}$.

Ci sono, poi, dei casi in cui un riesame della bibliografia e delle indicazioni di provenienza, reso possibile anche dal processo di digitalizzazione cui si stanno sottoponendo in questi anni le iscrizioni latine e greche di Roma e del resto del mondo antico, porta non all'aggiunta, ma all'espunzione di alcuni testi.

\footnotetext{
${ }^{21} \mathrm{Su}$ questi plutei e il loro reimpiego vd. A. Melucco Vaccaro, Corpus della scultura altomedievale, VII. La diocesi di Roma, III: la II regione ecclesiastica, Spoleto 1974, pp. 67-69, nr. 5, con foto a tav. II e, da ultimo, P. Pensabene, Roma su Roma. Reimpiego architettonico, recupero dell'antico e trasformazioni urbane tra il III e il XIII secolo, Città del Vaticano 2015, p. 445, con foto a p. 446, fig. 688 .

22 Ormai da scartare l'ipotesi di Büchler (CLE 1660) che, per la vicinanza con il Circo Massimo, proponeva di vedervi piuttosto l'elogio di un auriga, integrando il frammento CIL, VI 30364, 4 [--- Ph]erenico / [--- ces] sise $(t)$ habenis.

${ }^{23} \mathrm{Vd}$. in proposito la bibliografia raccolta e discussa da P. Pensabene, C. Panella, Reimpiego e progettazione architettonica nei monumenti tardo-antichi di Roma, II. Arco quadrifronte („Giano”) del Foro Boario, Rendiconti della Pontificia Accademia Romana di Archeologia 67, 1994-95, pp. 26-30 = AE 1997, 123; cfr. anche la più prudente posizione di F. Coarelli, Ianus Quadrifrons, [in:] LTUR, III, 1996, p. 94 e di C. Bariviera, Regione XI. Circus Maximus, [in:] A. Carandini (cur.), Atlante di Roma antica, Milano 2012, p. 437.

${ }^{24}$ Pubblicata da P. Mateos, A. Pizzo, A. Ventura, Arcus Divi Constantini: An Architectural Analysis and Chronological Proposal for the Arch of Janus in the Forum Boarium in Rome, Journal of Roman Studies 107, 2017, pp. 17-23, nr. A-3 (= EDR162832). Altri frammenti di iscrizioni imperiali reimpiegati nello stesso arco si trovano pubblicati nello stesso articolo, rispettivamente alle pp. 15-17, nr. A-1 (= EDR162830) e p. 17, nr. A-2 (= EDR162831).

${ }^{25}$ Secondo le convincenti argomentazioni sviluppate da P. Mateos, A. Pizzo, A. Ventura, Arcus Divi Constantini, pp. 23-31.
} 
L'iscrizione pubblicata da Géza Alföldy in CIL, VI 40627, quindi tra le urbane, sia pure di provenienza incerta, ad esempio, è in realtà prenestina ${ }^{26}$, mentre dalla città etrusca di Seperna proviene CIL, VI 40659, riconosciuta da Carlo Slavich come il testo già edito, tra le iscrizioni di Capena, in CIL, XI $3875^{27}$.

Ma il panorama delle novità relative alle iscrizioni imperiali di Roma non è fatto solo di „minima marginalia” destinati più a suscitare riflessioni di carattere metodologico che a rivoluzionare radicalmente il panorama dell'epigrafia urbana. Tanto interessante, quanto difficile da tracciare è anche il complesso dei nuovi studi relativi a iscrizioni già note, che ne hanno significativamente migliorato la comprensione e l'interpretazione negli ultimi anni. Nell'impossibilità di documentare tali apporti nella loro interezza, mi sia consentito di scegliere solo un paio di esempi particolarmente significativi, come la scoperta del frammento di una nuova copia, proveniente da Tifernum Tiberinum, del senatus consultum relativo agli onori funebri per Germanico ${ }^{28}$, che ha consentito importanti precisazioni alla lettura e all'integrazione della Tabula Siarensis e delle versioni urbane dello stesso testo ${ }^{29}$, o il recente studio dell'iscrizione relativa a un edificio pubblico costruito o restaurato da Traiano, reimpiegata nell'atrio di S. Clemente ${ }^{30}$ e convincentemente attribuita da Filippo Coarelli alla sede della Moneta ${ }^{31}$.

Ben diverso, naturalmente, il discorso relativo ai nuovi rinvenimenti veri e propri.

\section{NUOVE SCOPERTE}

Anche in questo caso, l'apporto di novità varia a seconda delle circostanze, e non dipende necessariamente né dalle dimensioni o dallo stato di conservazione del testo, né dal fatto che la scoperta sia stata fatta nel corso di nuovi scavi archeologici o di ricerche in archivio o in magazzino.

${ }^{26}$ Come già affermato da G. Barbieri, [in:] Il lapidario Zeri di Mentana, Roma 1982, pp. 57-58, nr. 21 = AE 1982, 146. Cfr. EDR078461 e EDR093021.

${ }^{27}$ Cfr. EDR144885.

${ }^{28}$ Pubblicato da M. Cipollone, Un frammento del senatus consultum de honoribus Germanici al Museo Archeologico di Perugia, Epigraphica 74, 2012, pp. 83-103 = AE 2012, 467 (= EDR132227).

${ }^{29}$ CIL, VI 911 = 31199, cfr. p. 4305 e CIL, VI 40349 (= EDR092841; EDR132295; EDR132297).

${ }^{30}$ CIL, VI 40492 = EDR092920.

${ }^{31}$ F. Coarelli, Argentum signatum. Le origini della moneta d'argento a Roma, Roma 2013, pp. 172-174, dove l'iscrizione è ancora citata nell'edizione di P. Lawlor, [in:] F. Guidobaldi, San Clemente. Gli edifici romani, la basilica paleocristiana e le fasi altomedievali, Roma 1992, pp. 329-332. 
Alcune delle novità più interessanti pubblicate negli ultimi anni, infatti, vengono dal ritrovamento o dal riconoscimento di nuovi frammenti pertinenti a iscrizioni già note, che costringono a modificare la lettura proposta in precedenza o, se anche la confermano, impongono comunque un ripensamento del testo e delle sue caratteristiche.

Alla prima categoria appartiene un frammento marmoreo rinvenuto nei pressi del Teatro di Marcello nel corso di indagini eseguite dalla Sovrintendenza capitolina nel 2000, e rimasto per molto tempo inedito. A mio avviso, infatti, è possibile riconoscervi la parte centrale dello stesso architrave cui appartiene un frammento già noto, pubblicato proprio nel Supplementum di Géza al numero CIL, VI 40317, dove, sia pure molto ipoteticamente, viene interpretato come iscrizione di dedica del teatro. Disponendo accuratamente nello spazio i due frammenti, solidali ma non contigui tra loro, è possibile proporre una nuova lettura del testo, da interpretare come dedica posta ad Augusto divinizzato da Livia - ormai Iulia Augusta - e Tiberio. L'anomala posizione dei nomi dei due dedicanti - prima Livia e poi Tiberio - troverebbe conferma in un passo di Tacito (Ann., III, 64) in cui, appunto, si ricorda che nel 22 fu posta, non procul a Theatro Marcelli, una dedica al Divo Augusto in cui Livia Tiberi nomen suo postscripserat ${ }^{2}$.

Conferma la lettura proposta sin dai tempi del Mommsen, invece, la scoperta di un nuovo frammento dell'iscrizione CIL, VI 966, avvenuta nel corso delle indagini preliminari per la costruzione della nuova linea C della Metropolitana di Roma. Il testo, in due esemplari, si ricostruisce completamente grazie a un grosso frammento pertinente alla parte centrale, rinvenuto tre secoli fa e conservato ai Musei Vaticani, a due frammenti noti da un disegno di Sallustio Peruzzi conservato nel Gabinetto Disegni e Stampe degli Uffizi di Firenze e, appunto, al nuovo frammento, pertinente all'angolo inferiore destro di una delle due copie ${ }^{33}$. La nuova scoperta, benché sia venuta sostanzialmente a confermare la lettura tradizionalmente accettata, è stata tuttavia l'occasione per riconsiderare il contesto da cui l'iscrizione proviene. Le dimensioni dell'architrave, tradizionalmente considerato la dedica del tempio dei Divi Traiano e Plotina, infatti, mal si accordano con le dimensioni eccezionali del tempio, confermate anche dalle recenti indagini archeologiche, e fanno pensare piuttosto alla monumentalizzazione di un passaggio del

\footnotetext{
${ }^{32}$ S. Orlandi, Le iscrizioni del Teatro, [in:] P. Ciancio Rossetto, G. Pisani Sartorio (cur.), Theatrum Marcelli, Roma 2017, pp. 188-191. Cfr. EDR092823.

${ }^{33}$ CIL, VI $966=31215=$ EDR103994.
} 
cortile che circondava la Colonna Traiana, ipotesi cui non osterebbe il tenore del testo ${ }^{34}$.

A una nuova, più corretta impaginazione del testo dell'iscrizione che doveva campeggiare sull'arco di Tito nel Circo Massimo, invece, ha portato la recentissima scoperta ${ }^{35}$ di alcuni frammenti dell'iscrizione in lettere metalliche pubblicata in CIL, VI 944 sulla base del solo apografo contenuto nella silloge di Einsiedeln ${ }^{36}$. I risultati della nuova ricostruzione grafica del testo, resa possibile dal fatto che due dei frammenti rinvenuti conservano parte della cornice inferiore e superiore dell'attico, sono stati presentati da Maria Grazia Granino nel corso di una giornata di studio dedicata ai nuovi scavi nell'area del Circo Massimo ${ }^{37}$, i cui atti sono in corso di pubblicazione.

Conferma la lettura già proposta in CIL, VI 40772 anche l'individuazione di un nuovo frammento dell'iscrizione monumentale relativa ad un restauro delle Terme di Caracalla in età costantiniana ${ }^{38}$ : è merito della Jenewein aver riconosciuto che il blocco era pertinente alla stessa trabeazione cui appartengono gli altri già pubblicati, ma tale scoperta non ha poi portato a una vera e propria riedizione del testo, per la quale si rinvia all'appendice di Giorgio Crimi in coda a questo studio.

Solo dalle fonti antiche era noto anche, fino ad alcuni anni fa, l'arco eretto sul Palatino da Augusto in onore del proprio padre naturale. L'edizione degli scavi eseguiti nel 1863 nell'area degli Orti Farnesiani, ha consentito a Maria Antonietta Tomei di individuare, tra gli appunti di Pietro Rosa, il riferimento ad alcuni frammenti pertinenti ad un arco, due dei quali iscritti, che sono poi stati rintracciati nello stesso luogo in cui erano stati visti e documentati oltre un secolo prima. La ricostruzione dell'arco e dell'iscrizione di dedica, originariamente in lettere di bronzo, posta sull'attico, ha permesso di identificare la porticus menzionata nel testo con il monumento dedicato

\footnotetext{
${ }^{34} \mathrm{Vd}$. in proposito le osservazioni di S. Orlandi, R. Egidi, Una nuova iscrizione monumentale dagli scavi di piazza Madonna di Loreto, Historikà 1, 2011, pp. 301-319 e, più recentemente, S. Orlandi, Le testimonianze epigrafiche, Bollettino di Archeologia, 4, 2013, pp. 52-59.

${ }^{35}$ Su cui vd. M. Buonfiglio, Circo Massimo. Scavi e restauri nell'edificio (2009-2015), Bullettino della Commissione Archeologica Comunale 115, 2014, pp. 326-338, in part. p. 332; S. Pergola, A. Coletta, Circo Massimo. Considerazioni sulla decorazione architettonica dell'Arco di Tito, ibidem, pp. 338-345, in part. p. 339.

${ }^{36}$ CIL, VI $944=$ EDR103960.

${ }^{37}$ Il Circo Massimo: scavi, indagini e ricostruzioni 2009-2106 (Roma, Auditorium dell'Ara Pacis, 9 maggio 2016). (F) 85 .

${ }^{38}$ G. Jenewein, Die Architekturdekoration der Caracallathermen, Wien 2008, p. 262, nr. A
} 
da Augusto nel 25 a.C. a C. Octavius, benché quest'ultimo non venga esplicitamente menzionato ${ }^{39}$.

Non da nuovi scavi, ma da una più attenta analisi di frammenti già editi, sia nel Corpus che in altre sedi, nasce, invece, il riesame della dedica delle Terme di Diocleziano e dei diversi esemplari in cui era redatta ${ }^{40}$ condotto a più riprese da Giorgio Crimi negli ultimi anni. All'individuazione di un frammento sicuramente pertinente a questa iscrizione, attualmente conservato nel Museo Archeologico di Alatri ${ }^{41}$, è seguito il riconoscimento di un altro frammento, pubblicato tra i frammenti conservati nell'Antiquarium Comunale del $\mathrm{Celio}^{42}$, e finora sfuggito all'attenzione degli studiosi ${ }^{43}$. Particolarmente interessante, in quest'ultimo caso, il fatto che già nell'addendum a p. 4327 Géza era giunto a proporre, pur non conoscendo il nuovo frammento, la stessa correzione di lettura ora confermata da questa recente scoperta.

Nel solco dell'esempio offerto da Alföldy con i suoi famosi studi sull'obelisco vaticano, l'acquedotto di Segovia e l'iscrizione del Colosseo si muove anche un recente studio di Ángel Ventura Villanueva che, se coglie nel vero, è destinato a rivoluzionare le nostre conoscenze sull'arco di Settimio Severo e le sue iscrizioni. Secondo questo studioso, infatti, alle iscrizioni con lettere alveolate presenti sui due lati dell'attico ${ }^{44}$, sarebbero andate ad aggiungersi, negli anni successivi alla dedica del monumento, una serie di dediche ai vari membri della famiglia imperiale divinizzati Divo Severo Pio ex s(enatus) c(onsulto), cui in seguito sarebbero state aggiunte, sui due lati, le dediche Divae Iuliae Aug(ustae) et Divo Anton(ino) Magno). Queste

${ }^{39}$ M.A. Tomei, I resti dell'arco di Ottavio sul Palatino e il portico delle Danaidi, Mélanges de l'Ecole Française de Rome. Antiquité 112, 2000, pp. 557-610 (= EDR112216). Per gli appunti del Rosa vd. già M.A. Tomei, Scavi francesi sul Palatino. Le indagini di Pietro Rosa per Napoleone III, Roma 1999, p. 332.

${ }^{40}$ CIL, VI $1130=31242=$ EDR110850 e EDR115769.

${ }^{41}$ G. Crimi, A. Cicogna, Dal centro di Roma alle campagne di Alatri: un „nuovo” frammento dell'iscrizione dedicatoria delle Terme di Diocleziano, Epigraphica 74, 2012, pp. 243-256 (= EDR129724).

${ }^{42}$ CIL, VI 30567, 20, a sua volta ripubblicato anche (senza che l'identità fosse riconosciuta) in CIL, VI 31463. Vd. in proposito G. Crimi, CIL VI, 30567, 20: una nota aggiuntiva, Epigraphica 78, 2016, pp. 384-388 (= EDR122748).

${ }_{43}$ G. Crimi, Il contributo di EDR all'aggiornamento del CIL: l'iscrizione dedicatoria delle Terme di Diocleziano alla luce di un nuovo frammento, Epigraphica 77, 2015, pp. 426-446; un'utile sintesi in G. Crimi, L'iscrizione dedicatoria delle Terme di Diocleziano, [in:] R. Friggeri, M. Magnani Cianetti (cur.), Le Terme di Diocleziano e la Certosa di Santa Maria degli Angeli, Roma 2014, pp. 57-67.

${ }^{44}$ CIL, VI $1033=$ EDR104093. 
iscrizioni aggiuntive sarebbero state composte con lettere metalliche applicate alla superficie del marmo e ricostruibili solo grazie ai fori lasciati dall'asportazione dei perni che le fissavano, tuttora visibili sulla fascia dell'architrave immediatamente al di sotto dell'iscrizione dell'attico ${ }^{45}$. La proposta è ancora troppo poco nota per essere entrata nel circuito delle pubblicazioni sull'età severiana, ma certamente non mancherà di far discutere la comunità degli studiosi, come già accadde, a suo tempo, per le varie proposte di ricostruzione delle epigrafi di cui rimangono solo le tracce dei perni.

A una giusta considerazione dello stretto rapporto che lega forma e contenuto, e in particolare l'adozione di particolari materiali e tecniche di scrittura e la rilevanza sociale dei personaggi menzionati nel testo dobbiamo anche il riconoscimento come epigrafi imperiali di una serie di frammenti marmorei con lettere alveolate pertinenti a più iscrizioni diverse, rinvenuti nel corso dei recenti scavi nella zona del tempio di Matidia e delle basiliche intitolate alla stessa Matidia e a Marciana, madre di quest'ultima e sorella di Traiano. Purtroppo, lo stato di conservazione dei frammenti non consente, in questo caso, di proporre una ricostruzione dei testi, ma l'altezza delle lettere interamente conservate $(\mathrm{cm} .18,5)$ sconsiglia di attribuire i frammenti all'iscrizione di dedica - che doveva essere ancora più monumentale - del tempio fatto costruire nel 119 da Adriano per la suocera ${ }^{46}$.

Allo stesso modo, l'uso delle litterae alveolatae (in questo verosimilmente destinate ad essere riempite non di metallo ma di una sostanza vischiosa come lo stucco o la pasta vitrea) unito al particolare materiale del supporto, il porfido, suggerisce di interpretare come resti di iscrizioni imperiali di una certa importanza anche i numerosi frammenti di epigrafi monumentali, solo in parte già pubblicati in altre sedi, raccolti in una recente monografia dedicata, appunto, all'uso del porfido nel mondo romano ${ }^{47}$. Se lo stato di

\footnotetext{
${ }^{45}$ A. Ventura Villanueva, L'arco di trionfo di Settimio Severo a Roma e le sue iscrizioni con litterae aureae. Una nuova prospettiva, Mitteilungen des Deutschen Archäologischen Institut. Römische Abteilung 120, 2014, pp. 267-312.

${ }^{46}$ Così l'editore dei frammenti, G.L. Gregori, [in:] F. Filippi (cur.), Campo Marzio. Nuove ricerche. Seminario di studi, Roma 2015, pp. 292-294; cfr. EDR158776; EDR158779; EDR158780; EDR158781. Degna di nota anche la menzione, in fonti del XV e XVI secolo, di „due iscrizioni monumentali di incerta lettura, recanti il nome di imperatori della dinastia antonina", rinvenute nella zona dell'attuale piazza Colonna e oggi perdute, individuate da A. Vella, ibidem, p. 185 con nota 47.

${ }^{47}$ D. Del Bufalo, Porphyry. Red Imperial Porphyry. Power and Religion, Turin 2012, pp. 208-210, nr. I 3-6 e 8, corrispondenti a EDR003014; EDR123614; EDR123615; EDR146333; EDR146344; EDR146572. Questi vanno ad aggiungersi al frammento già edito da Alföldy in CIL,
} 
conservazione del testo non ci viene molto in aiuto, la scelta di affidare all'uso di un particolare colore e di un particolare materiale una parte del messaggio che con le scritture esposte si intendeva trasmettere, conferma l'importanza degli elementi non verbali nella comunicazione epigrafica.

Come si vede, anche nei casi in cui la monumentale opera di Alföldy va aggiornata, corretta o integrata, aggiunte e correzioni devono molto, in realtà, alla metodologia inaugurata o perfezionata da questo studioso, soprattutto quando si tratta di integrare testi molto frammentari, per cui si cerca di ricavare il massimo delle informazioni possibili anche da poche lettere, o addirittura „inesistenti”, per i quali il lavoro dell'epigrafista rasenta quello dell'artista o dell'enigmista... Non sempre, però, i casi sono così disperati o eclatanti, e il sottosuolo di Roma sa essere, a volte, più generoso, restituendo testi di cui si conserva una porzione più consistente.

E' il caso, ad esempio, di una dedica posta dagli imperatori Marco Aurelio e Commodo su una stele in travertino rinvenuta lungo la via Laurentina ${ }^{48}$. Benché il testo sia molto laconico e lacunoso e non specifichi, almeno nella parte conservata, i lavori che furono oggetto dell'intervento imperiale, ritengo convincente la proposta di interpretarlo come iscrizione destinata ad essere inserita sul parapetto in laterizio del ponte a un'arcata che superava il fosso dell'Acqua Acetosa tra il V e il VI miglio della via Laurentina.

Di estremo interesse anche il blocco marmoreo iscritto conservato nel cortile di Palazzo Lancellotti ai Coronari, in cui Vincenza Morizio ha riconosciuto il resto di un'iscrizione monumentale, forse in origine esposta nel Mausoleo di Augusto, in cui erano ricordati gli onori posti in vestibulo domus eius da Caligola e dal senato in onore di Germanico e dei figli di questo, Nerone e Druso Cesari ${ }^{49}$.

VI 41044 (= EDR093329), e alla lastra, anch'essa molto frammentaria, inserita nel pavimento della chiesa di S. Maria in Aracoeli e pubblicata in CIL, VI 30323 (= EDR118355) che pure, a mio avviso, andrebbe recuperata alla sezione delle iscrizioni domus Augustae.

${ }^{48}$ Oggetto di una pubblicazione preliminare da parte di G.L. Gregori, [in:] M.A. Tomei (cur.), Roma. Memorie dal sottosuolo. Ritrovamenti archeologici 1980/2006, Milano 2006, p. 464, nr. II. 891 (= EDR033307).

${ }^{49} \mathrm{~A}$ parte l'interpretazione generale del testo, incerte rimangono le integrazioni delle parti mancanti: cfr. le ipotesi leggermente diverse proposte da V. Morizio, Una nuova iscrizione a Palazzo Lancellotti ai Coronari, [in:] M.L. Caldelli, G.L. Gregori, S. Orlandi (cur.), Epigrafia 2006. Atti della XIV $\mathrm{XV}^{\mathrm{e}}$ Rencontre sur l'épigraphie in onore di Silvio Panciera con altri contributi di colleghi, allievi e collaboratori, Roma 2008, pp. 339-348, e dalla stessa V. Morizio, [in:] Collezione di antichità di Palazzo Lancellotti ai Coronari, Roma 2008, pp. 241-243, nr. 83 (=EDR102221). 
Novità interessanti vengono anche da iscrizioni imperiali non del tutto inedite, ma che, benché pubblicate, o non sono state adeguatamente valorizzate, $\mathrm{o}$ addirittura non sono state riconosciute come tali.

Quasi sotto silenzio, ad esempio, è passata la scoperta, nel corso di lavori di ripulitura delle superfici marmoree della chiesa di Santa Prassede, di un architrave iscritto - evidentemente in reimpiego - che menziona lavori di restauro da parte di due imperatori i cui nomi sono andati perduti ma che si possono verosimilmente identificare con Settimio Severo e Caracalla ${ }^{50}$.

Alla dinastia dei Severi doveva appartenere anche il frammento con i cognomina ex virtute Arabicus e Adiabenicus, esposto nel museo didattico Radici del presente, recentemente allestito nel Palazzo delle Assicurazioni Generali in piazza Venezia. Di questo frammento, sfuggito finora a qualsiasi schedatura anche se sicuramente non di recente scoperta, il catalogo on line di questa esposizione costituisce la prima pubblicazione ${ }^{51}$.

Sempre in materia di pubblicazioni non del tutto adeguate alla rilevanza dei materiali, va registrato anche il caso di un frammento dall'Isola Tiberina pubblicato nel 2012 insieme ad altre iscrizioni con la stessa provenienza, che, per una svista redazionale, sono rimaste senza la relativa documentazione fotografica $^{52}$. Dovendo aggiornare il nostro archivio, abbiamo aggirato l'ostacolo facendoci mandare direttamente dagli autori le foto che avrebbero dovuto accompagnare l'edizione dei pezzi, e ci siamo così resi conto che uno di questi è verosimilmente parte di un'iscrizione imperiale, come suggerisce l'uso di litterae alveolatae che, a partire dall'età augustea, diventa una prerogativa pressoché esclusiva dell'imperatore e dei membri della sua famiglia.

Non va dimenticata, infine, la messe di novità che può continuare ad arrivare non da scavi archeologici o a campagne di schedatura dei materiali epigrafici, ma da indagini tra le carte degli archivi e dei manoscritti.

\footnotetext{
${ }^{50}$ Del testo si conserva ancora solo la sequenza [--- p]ius felix Aug(ustus) refecerun[ $t$----], che però, come suggeriscono numerosi confronti, si può integrare con il nome di Caracalla [--- M(arcus) Aurel(l)ius Antoninus p]ius felix ecc. (meno probabile l'integrazione del nome di Gordiano). Per l'analisi stilistica dell'architrave iscritto vd. P. Pensabene, Provenienze e modalità di spogliazione e reimpiego a Roma tra tardoantico e medioevo, [in:] Marmoribus vestita. Miscellanea in onore di Federico Guidobaldi, Città del Vaticano 2011, pp. 1080-1081 e idem, Roma su Roma. Reimpiego architettonico, recupero dell'antico e trasformazioni urbane tra il III e il XIII secolo, Città del Vaticano 2015, p. 426, con foto a p. 415, fig. 602 (= EDR162616).

${ }^{51}$ [online]: <www.radicidelpresente.it>, E 37 (= EDR137430). Sulla collezione epigrafica di cui il pezzo fa parte vd. C. Ferro, I. Franco, [in:] M. Bertinetti (cur.), Supplementa Italica - Imagines, Roma (CIL, VI) 5. Collezioni urbane dei palazzi storici, Roma 2016, pp. 286-287.

${ }^{52}$ W.N. Bruce, R.S. Wagman, Frustula tiberina. Epigraphical Fragments from the Tiber Island and Environs, Epigraphica 74, 2012, pp. 374-378, in part. pp. 377-378, nr. 9 (= EDR154287).
} 
Un più attento esame dell'articolo di Francesca De Capraris e della documentazione d'archivio su cui si basa, ad esempio, hanno permesso di individuare l'omissione di un pezzo nell'edizione dei frammenti attribuibili ad un non meglio identificato monumentum Claudianum pubblicati in CIL, VI 40420-40430. Confrontando, infatti, l'edizione del Corpus e il foglio dell'archivio Gatti che documenta il materiale epigrafico rinvenuto nello stesso contesto, Dimosthenis Kosmopoulos ha potuto constatare come manchi all'appello uno dei frammenti, attualmente irreperibile, ma ben documentato grazie ad un calco su carta velina eseguito al momento della scoperta e tuttora conservato nell'Archivio Storico della Soprintendenza Archeologica di Roma a Palazzo Altemps ${ }^{53}$.

Infine, è grazie all'esame di un manoscritto conservato nell'archivio della cattedrale di Girona, in Spagna, compilato tra il 1475 e il 1510 da padre Miquel Carbonell che è stato possibile ritrovare un gruppo di iscrizioni inedite di Roma, viste nel XV secolo sul retro del Palazzo Apostolico presso la basilica di S. Pietro in Vaticano e poi evidentemente andate perdute. Tra queste, oltre a materiali provenienti verosimilmente dalla necropoli della via Tiumphalis, si distingue per importanza una dedica a Massenzio, che va ad aggiungersi alle non molte iscrizioni urbane relative a questo imperatore $^{54}$. A giudicare dal formulario e dalla disposizione del testo (non accompagnato - come è normale nelle sillogi epigrafiche quattrocentesche - da un disegno o da una descrizione del supporto), potrebbe essere uno dei numerosi miliari massenziani rinvenuti in varie regioni d'Italia, dai quali, però, si distinguerebbe per la presenza degli epiteti clementissimo et invictissimo che precedono la consueta titolatura Domino nostro[Imp(eratori) Caes(ari)?] M(arco) Aur(elio) Valerio Maxentio semper Augusto. Tuttavia, la consapevolezza che la trascrizione non è frutto di un'autopsia diretta ma dipende da altre copie, a loro volta perdute ${ }^{55}$, lascia ampi margini di incertezza all'esatta trascrizione e interpretazione del testo.

${ }^{53}$ D. Kosmopoulos, EDR - Effetti collaterali 2, Scienze dell'Antichità 21.1, 2015, pp. 250-252, nr. 07 (= EDR162801).

${ }^{54}$ J. Carbonell Manils, Inscripciones inéditas del Phrygianum y de las necrópolis vaticanas (Girona, Arxiu de la Catedral, MS 69 de Pere Miquel Carbonell), Zeitschrift für Papyrologie und Epigraphik 194, 2015, p. 263, nr. 6 (= EDR150916).

${ }_{55} \mathrm{Ibidem}$, pp. $269-270$ propone, sia pure ipoteticamente, di riconoscere la fonte di padre Carbonell nel prelato catalano Jeroni Pau, che fu a Roma, a stretto contatto con gli ambienti della cancelleria apostolica, tra il 1475 e il 1492, e che potrebbe aver copiato le iscrizioni de visu, ma anche da altre sillogi precedenti come quella di Ciriaco d'Ancona. 
Casi come quelli fin qui ricordati dimostrano quanto resti ancora da fare non solo grazie alle sempre nuove scoperte che il sottosuolo di Roma ci continua a regalare, ma anche dal semplice riesame dei numerosissimi frammenti, spesso ancora inediti, che si conservano - in modo più o meno adeguato e facilmente accessibile - nei locali e nei magazzini dei vari musei, collezioni, soprintendenze e archivi da cui le antichità di Roma dipendono. E’ grazie a questa pressoché inesauribile ricchezza che possiamo sempre mantenere viva la speranza che anche problemi epigrafici apparentemente „impossibili” trovino prima o poi uno spiraglio di luce per essere chiariti o risolti.

E' proprio con due di questi „problemi epigrafici” che vorrei chiudere la mia rassegna. Nel volume del Supplementum, infatti, sono presenti due numeri - CIL, VI 40599 e 40689 - che si presentano in modo singolare, perché a una sommaria descrizione dell'argomento non corrisponde alcun testo.

Nel caso del titulus incerti generis imperatorum pubblicato come CIL, VI 40689 (= EDR125310) si tratta, in realtà, di un misunderstanding. Nel Nuovo Bollettino di Archeologia Cristiana del 1920, infatti, il Marucchi parla della presenza, nelle catacombe di S. Sebastiano, dei tre nomi di Gordiano, Pupieno e Balbino che ricordano personaggi contemporanei dei tre imperatori omonimi. Da questa notizia Alföldy ha ipotizzato la presenza, in queste catacombe, di un'iscrizione imperiale, forse in frammenti, che però non era riuscito a trovare né in catacomba, né nello schedario della Pontificia Commissione di Archeologia Sacra. In realtà l'iscrizione c'è, ma non ha né la forma, né la posizione, né il contenuto che Géza aveva evidentemente immaginato. Si tratta infatti di un gruppo di tre iscrizioni, in lingua latina, ma in caratteri greci, dipinte sulle volte degli ambienti più interni di uno dei sepolcri della cosiddetta piazzola, il mausoleo Y, e documentano una fase d'uso del sepolcro nella prima metà del III secolo da parte di un collegium funeraticium, quello degli Innocentiores, alcuni membri del quale portano, significativamente, i nomi di Pupieno, Balbino e Gordiano, ma chiaramente non sono imperatori ${ }^{56}$.

\footnotetext{
${ }^{56}$ Come risulta chiaramente dall'edizione di G. Mancini, Scavi sotto la basilica di S. Sebastiano sull'Appia Antica. Notizie degli Scavi di Antichità, 1923, pp. 65, 68-69, ripresa da F. FeraudiGruénais, Inschriften und Selbstdarstellung in stadtrömischen Grabbauten, Roma 2003, pp. 107108, nr. 146-148, e da A.M. Nieddu, Innocentiorum monumentum, [in:] LTUR, Suburbium, III, 2005, pp. 84-87.
} 
Più felice il caso di „un grande frammento di trabeazione” rinvenuto in pieno Campo Marzio, tra via del Pie' di Marmo e via S. Stefano del Cacco. Le informazioni che si ricavano dalla pagina di Notizie degli Scavi del 1925 sono state pubblicate nell'unico modo possibile sotto il numero CIL, VI 40599 (= EDR125295) con l'indicazione della presenza dei nomi di Settimio Severo e Caracalla e la constatazione che "non si è potuto copiare a causa della posizione dei due pezzi” e che l'iscrizione tuttora "latet sine dubio ibidem sub terra". Apparentemente un caso senza speranza, reso più grave e frustrante dall'evidente imponenza del monumento cui l'iscrizione doveva appartenere. Ma qualche volta la fortuna premia il tempo e la pazienza che si dedicano alla ricerca. E così è stato per questo blocco, riconosciuto in un taccuino di Edoardo Gatti conservato nell'Archivio Centrale dello Stato, da cui si riesce a recuperare il testo dell'iscrizione, incisa su due diverse fasce di un architrave che corrispondono ad altrettante fasi di scrittura: una da cui si ricava un intervento sul templum Sarapidis da parte di Caracalla, ricordato in nominativo (verosimilmente insieme al padre Settimio Severo) con la titolatura completa che comprendeva la menzione degli antenati fino al Divo Traiano, e un'altra, nella fascia sottostante, che documenta un restauro dello stesso edificio ad opera di Diocleziano e Massimiano ${ }^{57}$.

Tutta questa serie di novità, aggiunte, correzioni che si sono accumulate in un arco di tempo relativamente breve, meno di 25 anni, ci devono indurre innanzi tutto a una constatazione, e cioè alla necessità di considerare ogni risultato scientifico ed editoriale come un traguardo, sì, ma provvisorio, destinato inevitabilmente ad essere superato dal progredire delle scoperte e degli studi. In queste condizioni, sorge spontanea una domanda - che infatti molti miei studenti, nella loro spontaneità, mi fanno: operazioni come la raccolta e l'edizione dei testi nei volumi del Corpus Inscriptionum Latinarum conservano un significato anche se sappiamo in partenza che prima o poi dati e commenti fatalmente invecchiano e devono essere aggiornati? La risposta naturalmente è sì, perché un momento e uno strumento che faccia periodicamente il punto della situazione è e resta indispensabile, qualunque sia l'aspetto che il CIL prenderà nel prossimo futuro. Ma al tempo stesso è innegabile che noi studiosi dobbiamo attrezzarci, sia spiritualmente che materialmente, perché un costante aggiornamento dei dati possa avvenire nel

\footnotetext{
${ }^{57}$ L'appunto è stato pubblicato in Scienze dell'Antichità 23.1, 2017, pp. 135-148, in uno studio congiunto da parte di M. Giovagnoli e A. Ten, che inserisce questo testo nell'analisi dell'Iseo Campense e del suo contesto topografico sulla base delle evidenze archeologiche tuttora rilevabili, delle informazioni fornite dalle fonti antiche e dei documenti d'archivio.
} 
modo più semplice e rapido possibile. In questo, la tecnologia e gli strumenti di condivisione della conoscenza che si stanno evolvendo negli ultimi anni anche nel campo dell'epigrafia possono, se correttamente utilizzati nelle loro indubbie potenzialità, aiutarci. Strumenti non sostitutivi ma complementari delle grandi imprese editoriali sono ormai diventati le grandi banche dati epigrafiche - la Epigraphisces Datenbank Clauss Slaby (<http://www. manfredclauss.de $>$ ), per la sezione del Corpus che qui ci interessa in particolare l'Epigraphic Database Roma (<www.edr-edr.it $>$ ), senza dimenticare gli aggiornamenti bibliografici dell'Archivium Corporis Electronicum: (<http://cil.bbaw.de/cil_en/dateien/datenbank_eng.php $>$ ) e i progetti che le aggregano, come il portale di EAGLE (Europeana network of Ancient Greek and Latin Epigraphy: <www.eagle-network.eu>), che sta lavorando per rendere contemporaneamente consultabili numerosi archivi on line. Sta a noi, quindi, avere la capacità di lavorare su più piani, mantenendo il rigore scientifico che l'edizione critica di un testo epigrafico richiede e presuppone, ma non trascurando al tempo stesso la necessità di condividere immediatamente queste conoscenze con l'intera comunità scientifica. Con i mezzi che l'informatica umanistica e internet mettono a nostra disposizione, ma senza dimenticare generosità e intelligenza, perché anche con le pietre del passato possiamo camminare verso il futuro.

\section{APPENDICE}

\section{Giorgio Crimi (Roma)}

\section{UN FRAMMENTO POCO NOTO DELL'ISCRIZIONE COSTANTINIANA DELLE TERME DI CARACALLA}

Il complesso delle Terme di Caracalla, inaugurato nel 216 e ultimato nel 235 d.C..$^{58}$, fu oggetto di un intervento di restauro in età costantiniana ${ }^{59}$ :

\footnotetext{
${ }^{58}$ Per una disamina completa delle fonti letterarie riguardanti la storia delle Terme di Caracalla vd. M. Piranomonte, Thermae Antoninianae, [in:] LTUR, V, 2000, pp. 42-48, cui si aggiunga M. Piranomonte, Nuovi dati sulle Terme di Caracalla, [in:] D. Manacorda, R. Santangeli Valenzani (cur.), Il primo miglio della via Appia a Roma, Roma 2011, pp. 53-59 per i nuovi dati archeologici.

${ }^{59}$ D. DeForest, Emperors, baths, and public space: the imperial thermae in Rome's late antique landscape, [in:] G. Smith, J. Gadeyne, Perspectives on public space in Rome, from antiquity to the present day, Burlington 2013, p. 51; per la titolatura di Costantino vd. A. Filippini, G.L. Gregori,
} 
un'arcata del calidarium fu sostituita da un'abside, connessa ad una trabeazione curvilinea con iscrizione dedicatoria disposta su tre registri, che presenta lettere capitali eseguite nel complesso accuratamente e, infine, rubricate $^{60}$. Di questa trabeazione, rinvenuta in un'area prossima al calidarium stesso e conservata nei sotterranei del complesso termale, sono noti soprattutto due grandi blocchi marmorei iscritti non contigui fra loro. L'edizione più aggiornata è quella fornita da Andrea Scheithauer in CIL VI $40772^{61}$, il cui lemma descrittivo si conclude con una precisazione: „accessui clausum videre non potuit et ad im. phot. et ex Panciera, sched. descripsit Alföldy a. 1989”62. Nell'archivio di Epigrafia Latina della Sapienza Università di Roma, tuttavia, si conserva la foto di un ulteriore frammento di questa iscrizione: esso misura $\mathrm{cm} 35 \times 65 \times 60$, è pertinente alla prima riga di testo e vi sono incise cinque lettere, parzialmente conservate, che vanno ad integrare il nome di Costantino $^{63}$ (figg. 1-2). Il frammento è già stato riconosciuto come pertinente alla stassa trabeazione dal punto di vista archeologico, ma non è mai stato riconsiderato dal punto di vista epigrafico ${ }^{64}$. Grazie all'individuazione del „nuovo” frammento e al controllo autoptico di tutti i blocchi iscritti ${ }^{65}$, si può ora proporre una nuova trascrizione, che aggiorna quella presente in CIL, VI 40772:

L'epigrafia costantiniana. La figura di Costantino e la propaganda imperiale, [in:] Costantino I. Enciclopedia costantiniana sulla figura e l'immagine dell'imperatore del cosiddetto editto di Milano, 313-2013, Roma 2013, pp. 517-541.

${ }^{60}$ Una foto a colori in cui si apprezza la rubricatura delle lettere, seppur limitatamente al frammento destro della trabeazione, è presente in M. Piranomonte, Terme di Caracalla, Milano 2008, p. 9.

${ }^{61}$ Cfr. EDR073541.

${ }^{62}$ Ai sotterranei delle Terme di Caracalla aveva avuto accesso, invece, il regista Luigi Magni per le riprese del film „Nell'anno del Signore”, uscito nelle sale cinematografiche nel 1969. Nella scena in cui l'attore Ugo Tognazzi recita la parte del cardinale Agostino Rivarola è chiaramente visibile la porzione destra dell'iscrizione in questione utilizzata come piano scrittorio, per mettere agli atti, dopo un processo sommario, la condanna alla ghigliottina di due affiliati alla carboneria (ringrazio la prof.ssa Silvia Orlandi per avermi segnalato la scena del film, per la quale si vd. [online], YouTube [accesso: 2017-05-15]. Disponibile a: <https://www.youtube.com/watch?v=XSl0iNpXTw>.

${ }^{63}$ Anche questo frammento si conserva nei sotterranei delle Terme di Carcalla.

${ }^{64}$ G. Jenewein, Die Architekturdekoration der Caracallathermen, Wien 2008, pp. 167-168 e 262-263.

${ }^{65}$ Un confronto tra i due blocchi maggiori della trabeazione permette di scorgere alla seconda linea di testo una sensibile differenza qualitativa a livello paleografico (c'è una distanza di $3 \mathrm{~cm}$ e $5 \mathrm{~cm}$ tra una lettera e l'altra rispettivamente nel blocco sinistro e nel blocco destro). Sebbene il blocco sinistro presenti lettere non conservate in tutta la loro altezza, emerge in maniera evidente che esse, rispetto a quelle incise sul blocco destro, sono eseguite con minore accuratezza e risultano molto ravvicinate tra loro, mentre nel blocco destro hanno maggiore spaziatura. Da una visione 


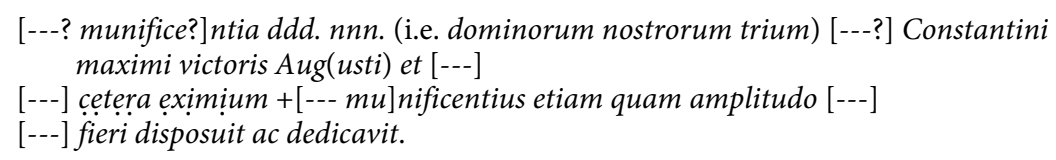

La parola mutila all'inizio del testo è stata in passato integrata in vari modi. Sebbene esista effettivamente la possibilità che in origine vi fosse scritto munificentia alla prima riga e munificentius nella riga sottostante ${ }^{66}$, mi chiedo se non si possa pensare ad integrazioni alternative a questa, come suggerisce il confronto con altre iscrizioni di età costantiniana relative ad opere pubbliche: non escluderei, quindi, l'integrazione di termini come indulgentia o providentia ${ }^{67}$, magnificentia ${ }^{68}$ e clementia ${ }^{69}$.

Un altro punto importante riguarda la titolatura di Costantino: dal momento che il suo nome completo era Flavius Valerius Constantinus, è possibile che oltre al frammento in questione esistessero altri frammenti contenenti almeno uno dei due elementi onomastici ${ }^{70}$. Infatti, sebbene non sia stato possibile accostare il nuovo frammento ai blocchi già noti, è visibile in frattura sul lato destro il braccio sinistro della lettera $T$ che si congiunge al resto, mentre non altrettanto si può dire della parte sinistra del frammento: alla prima riga l'ultima $N$ è seguita a distanza di $15 \mathrm{~cm}$ ca da un segno di interpunzione e altrettanto spazio occorre immaginare prima dell'inizio del nome di Costantino.

Per quanto riguarda il resto del testo, viene ricordata la presenza di tre personaggi dei quali il primo è senza dubbio Costantino I, menzionato con l'epiteto victor che colloca l'iscrizione dopo la vittoria su Licinio del 324, che permise a Costantino di rimanere unico imperatore ${ }^{71}$. Gli altri dovevano essere i due figli Costantino II e Costanzo II, cosa che consente di fissare il terminus post quem al 326, anno in cui fu giustiziato Crispo, che nell'iscrizione - relativa solo a tre membri della famiglia imperiale - non

ravvicinata non ho però riscontrato erasioni o interventi che inducano a credere una rilavorazione della pietra in quel punto.

${ }^{66}$ Secondo la proposta avanzata da H. Fuhrmann, Archäologische Anzeiger, 1941, col. 522 e accolta anche da T. Grünewald, Constantinus Maximus Augustus. Herrschaftspropaganda in der zeitgenössischen Überlieferung, Stuttgart 1990, p. 220, nr. 262.

${ }^{67}$ Come proponeva già A. Degrassi, Epigrafia romana - I. Roma (1937-46), Doxa 2, 1949, p. 83 = A. Degrassi, Scritti vari di antichità, I, Roma 1962, p. 355. Cfr. AE 2001, $1009=$ EDR007172 (da Aquileia).

${ }^{68}$ Cfr. AE 1939, $151=$ AE 1983, $194=$ EDR073395 (da Abellinum).

${ }^{69}$ Cfr. CIL, VI 40770a-b = EDR075982 e EDR118900 (da Ostia).

${ }^{70}$ A. Filippini, G.L. Gregori, L'epigrafia costantiniana, p. 519, con appendice epigrafica alle pp. 526-531.

${ }^{71}$ Ibidem, p. 518. 
doveva comparire, mentre il terminus ante quem è costituito dal 333, anno in cui fu designato Cesare anche Costante.

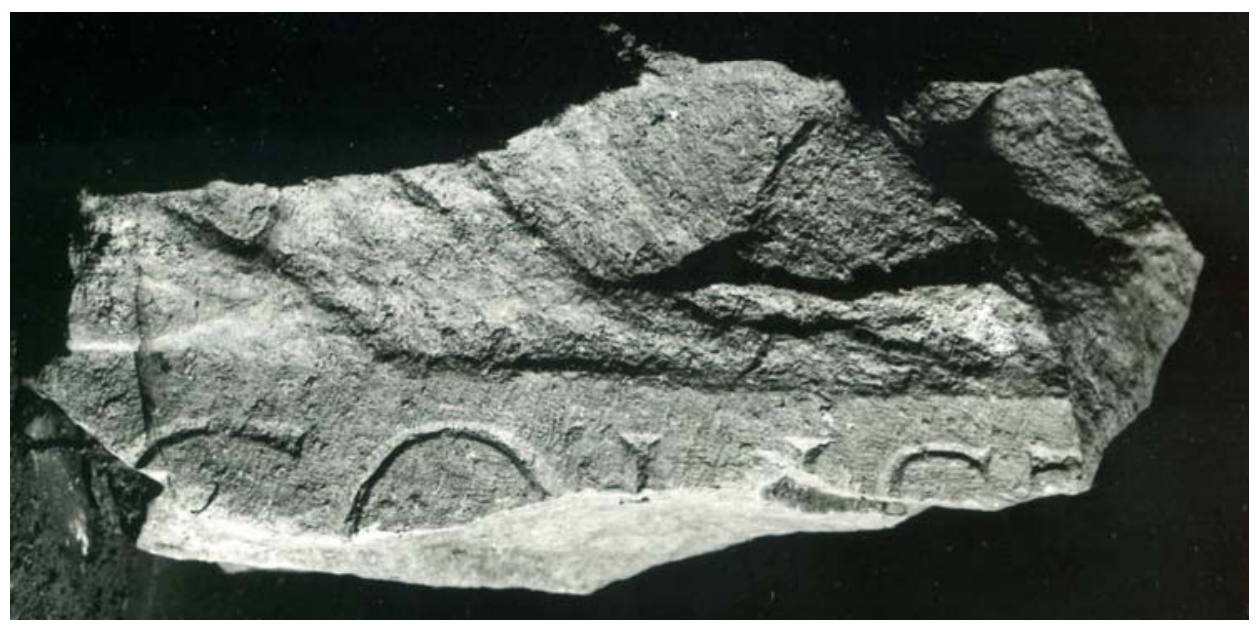

Fig. 1. Frammento inedito dell'iscrizione costantiniana delle Terme di Caracalla. Neg. Sapienza Università di Roma 11656. Su concessione del Ministero dei beni e delle attività culturali e del turismo - Soprintendenza Speciale per il Colosseo e l'Area archeologica centrale di Roma

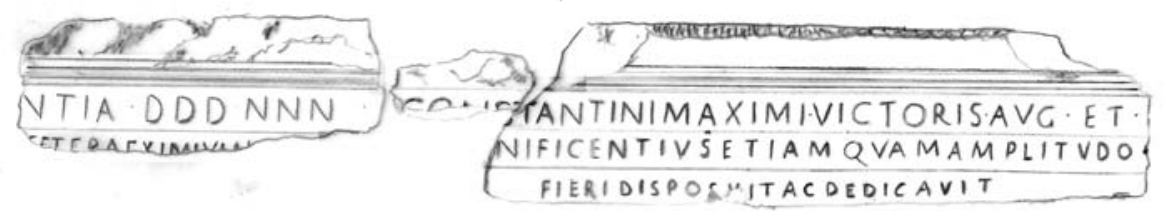

Fig. 2. Ricostruzione grafica dell'iscrizione costantiniana delle Terme di Caracalla. Disegno di Giorgio Crimi 


\section{A SUPPLEMENT TO A SUPPLEMENT: IMPERIAL INSCRIPTIONS FROM ROME FROM THE 1990S UNTIL THE PRESENT DAY}

\section{Summary}

After the publication of the last fascicle of the VI volume of CIL, in 1933, it took more than 60 years to have a new Supplement for the imperial inscriptions of Rome, published by Géza Alföldy in 1996. But in about 20 years, a lot of new inscriptions, or new fragments of old inscripitons, have been found, studied and edited, so that the last Supplement already needs to be updated.

Aim of this paper is to collect not the whole number, but a large selection of new discoveries and new studies that have changed the scenario of the imperial inscriptions from Rome, including the recognition of neglected fragments as important building inscripitons (as in the case of CIL, VI 30681, 1 - from the Theatre of Marcellus, 30364 - from the so called „Arco di Giano” - and 30567, 20 from the Diocletian's Baths).

New excavations in the centre of Rome led also to important discoveries related to big monuments like, for example, the Tajan's Forum, with a new interpretation of the inscription traditionally attributed to the temple of the Divi Trajan and Plotina (CIL, VI 966), or the Circus Maximus, where there were found fragments of the inscribed arch until now known only from the Einsideln epigraphic manuscript (CIL, VI 944).

Not less important the discoveries made possible by researches in public and ecclesiastic archives, that led to the knowledge of documents and fragments now lost, and in private buildings of Rome, where important inscriptions, thought to be lost, are still preserved.

As a whole, this study leads us to reflect on the important role of editorial enterprises like the Corpus Inscriptionum Latinarum, but at the same time, on the need to keep these research tools constantly updated through digital projects like the major epigraphic databases (Epigraphisches Datenbank Clauss Slaby; Epigraphic Database Roma; Europeana network of Ancient Greek and Latin Epigraphy).

\section{Bibliografia}

Ambrosino R., Riferimenti all'ordinamento associativo romano, Bullettino della Commissione Archeologica Comunale 67, 1939, pp. 85-99.

Barbanera M., Freccero A. (cur.), Collezione di antichità di Palazzo Lancellotti ai Coronari. Archeologia, Architettura, Restauro, Roma 2008

Barbieri G. (cur.), Il lapidario Zeri di Mentana, Roma 1982. 
Bariviera C., Regione XI. Circus Maximus, [in:] A. Carandini (cur.), Atlante di Roma antica, Milano 2012, pp. 421-445.

Bertinetti M. (cur.), Supplementa Italica - Imagines, Roma (CIL, VI) 5. Collezioni urbane dei palazzi storici, Roma 2016.

Bruce W.N., Wagman R.S., Frustula tiberina. Epigraphical Fragments from the Tiber Island and Environs, Epigraphica 74, 2012, pp. 374-378.

Buonfiglio M., Circo Massimo. Scavi e restauri nell'edificio (2009-2015), Bullettino della Commissione Archeologica Comunale 115, 2014, pp. 326-338.

Carbonell Manils J., Inscripciones inéditas del Phrygianum y de las necrópolis vaticanas (Girona, Arxiu de la Catedral, MS 69 de Pere Miquel Carbonell), Zeitschrift für Papyrologie und Epigraphik 194, 2015, pp. 259-270.

Chioffi L., Epigrafi tra Roma e Anzio: note a margine, Rendiconti della Pontificia Accademia Romana di Archeologia 88, 2015-2016, pp. 424-442.

Cipollone M., Un frammento del senatus consultum de honoribus Germanici al Museo Archeologico di Perugia, Epigraphica 74, 2012, pp. 83-103.

Coarelli F., Ianus Quadrifrons, [in:] LTUR, III, 1996, p. 94.

Coarelli F., Argentum signatum. Le origini della moneta d'argento a Roma, Roma 2013.

Crimi G., L'iscrizione dedicatoria delle Terme di Diocleziano, [in:] R. Friggeri, M. Magnani Cianetti (cur.), Le Terme di Diocleziano e la Certosa di Santa Maria degli Angeli, Roma 2014, pp. 57-67.

Crimi G., Il contributo di EDR all'aggiornamento del CIL: l'iscrizione dedicatoria delle Terme di Diocleziano alla luce di un nuovo frammento, Epigraphica 77, 2015, pp. 426-446.

Crimi G., CIL VI, 30567, 20: una nota aggiuntiva, Epigraphica 78, 2016, pp. 384-388.

Crimi G., Cicogna A., Dal centro di Roma alle campagne di Alatri: un „nuovo” frammento dell'iscrizione dedicatoria delle Terme di Diocleziano, Epigraphica 74, 2012, pp. 243-256.

DeForest D., Emperors, baths, and public space: the imperial thermae in Rome's late antique landscape, [in:] G. Smith, J. Gadeyne (cur.), Perspectives on public space in Rome, from antiquity to the present day, Burlington 2013, pp. 43-64.

Degrassi A., Epigrafia romana - I. Roma (1937-46), Doxa 2, 1949, pp. 47-135.

Degrassi A., Scritti vari di antichità, I, Roma 1962.

Del Bufalo D., Porphyry. Red Imperial Porphyry. Power and Religion, Turin 2012.

Feraudi-Gruénais F., Inschriften und Selbstdarstellung in stadtrömischen Grabbauten, Roma 2003.

Filippini A., Gregori G.L., L'epigrafia costantiniana. La figura di Costantino e la propaganda imperiale, [in:] Costantino I. Enciclopedia costantiniana sulla figura e l'immagine dell'imperatore del cosiddetto editto di Milano, 313-2013, Roma 2013, pp. 517-54.

Giovagnoli M., Ten A., Il tempio di Serapide in Campo Marzio: nuovi dati da un'iscrizione inedita, Scienze dell'Antichità 23.1, 2017, pp. 135-148.

Gregori G.L., „Tempio di Matidia”. Materiali epigrafici, [in:] F. Filippi (cur.), Campo Marzio. Nuove ricerche. Seminario di studi, Roma 2015, pp. 292-294.

Grünewald T., Constantinus Maximus Augustus. Herrschaftspropaganda in der zeitgenössischen Überlieferung, Stuttgart 1990.

Guidobaldi F., San Clemente. Gli edifici romani, la basilica paleocristiana e le fasi altomedievali, Roma 1992.

Jenewein G., Die Architekturdekoration der Caracallathermen, Wien 2008.

Marchionni R., La tradizione non solo manoscritta del Carmen epigraphicum patris opus munusque suum (CIL VI 1163). I segreti dell'obelisco lateranense, Rendiconti della Pontificia Accademia Romana di Archeologia 85, 2012-2013, pp. 455-472.

Mateos P., Pizzo A., Ventura A., Arcus Divi Constantini: An Architectural Analysis and Chronological Proposal for the Arch of Janus in the Forum Boarium in Rome, Journal of Roman Studies 107, 2017, pp. 1-38.

Mekacher N., Die vestalischen Jungfrauen in der römischen Kaiserzeit, Wiesbaden 2006. 
Melucco Vaccaro A., Corpus della scultura altomedievale, VII. La diocesi di Roma, III: la II regione ecclesiastica, Spoleto 1974.

Monterroso A., La scenae frons ne los teatros de Roma. Entre liturgia, formas y modelos, [in:] La scenae frons en la arquitectura teatral romana, Cartagena 2009, pp. 15-55.

Morizio V., Una nuova iscrizione a Palazzo Lancellotti ai Coronari, [in:] M.L. Caldelli, G.L. Gregori, S. Orlandi (cur.), Epigrafia 2006. Atti della XIV ${ }^{e}$ Rencontre sur l'épigraphie in onore di Silvio Panciera con altri contributi di colleghi, allievi e collaboratori, Roma 2008, pp. 339-348.

Morselli C., Tortorici E., Curia, Forum Iulium, Forum Transitorium, Roma 1989.

Nieddu A.M., Innocentiorum monumentum, [in:] LTUR, Suburbium, III, 2005, pp. 84-87.

Orlandi S., Un'iscrizione monumentale dall'area del Teatro di Marcello, [in:] G. Paci (cur.), Contributi all'epigrafia di età augustea. Actes de la XIII ${ }^{e}$ Rencontre franco-italienne sur l'epigraphie di monde romain, Tivoli 2007, pp. 201-216.

Orlandi S., Le testimonianze epigrafiche, Bollettino di Archeologia on line, 4, 2013, pp. 45-59.

Orlandi S., Le iscrizioni del Teatro, [in:] P. Ciancio Rossetto, G. Pisani Sartorio (cur.), Theatrum Marcelli, Roma 2017, pp. 185-191.

Orlandi S., Egidi R., Una nuova iscrizione monumentale dagli scavi di piazza Madonna di Loreto, Historikà 1, 2011, pp. 301-319.

Orlandi S., Carapellucci A., D’Andrea F., Gabrielli I., Kosmopoulos D., Martino C., Serra M., EDR - Effetti collaterali 2, Scienze dell'Antichità 21.1, 2015, pp. 241-254.

Panciera S. (cur.), Iscrizioni greche e latine del Foro Romano e del Palatino, Tituli, 7, Roma 1996.

Panciera S., Epigrafi, epigrafia, epigrafisti. Scritti vari editi e inediti (1956-2005) con note complementari e indici, Vetera, 16, Roma 2006.

Pensabene P., Provenienze e modalità di spogliazione e reimpiego a Roma tra tardoantico e medioevo, [in:] Marmoribus vestita. Miscellanea in onore di Federico Guidobaldi, Città del Vaticano 2011, pp. 1049-1088.

Pensabene P., Roma su Roma. Reimpiego architettonico, recupero dell'antico e trasformazioni urbane tra il III e il XIII secolo, Città del Vaticano 2015.

Pensabene P., Panella C., Reimpiego e progettazione architettonica nei monumenti tardoantichi di Roma, II. Arco quadrifronte („Giano”) del Foro Boario, Rendiconti della Pontificia Accademia Romana di Archeologia 67, 1994-95, pp. 25-67.

Pergola S., Coletta A., Circo Massimo. Considerazioni sulla decorazione architettonica dell'Arco di Tito, Bullettino della Commissione Archeologica Comunale 115, 2014, pp. 338-345.

Piranomonte M., Thermae Antoninianae, [in:] LTUR, V, 2000, pp. 42-48.

Piranomonte M., Terme di Caracalla, Milano 2008.

PiranomonteM., Nuovi dati sulle Terme di Caracalla, [in:] D. Manacorda, R. Santangeli Valenzani (cur.), Il primo miglio della via Appia a Roma, Roma 2011, pp. 53-59.

Tomei M.A., Scavi francesi sul Palatino. Le indagini di Pietro Rosa per Napoleone III, Roma 1999.

Tomei M.A., I resti dell'arco di Ottavio sul Palatino e il portico delle Danaidi, Mélanges de l'Ecole Française de Rome. Antiquité 112, 2000, pp. 557-610.

Tomei M.A. (cur.), Roma. Memorie dal sottosuolo. Ritrovamenti archeologici 1980/2006, Milano 2006.

Vella A., Tempio di Adriano. Nuovi dati, [in:] F. Filippi (cur.), Campo Marzio. Nuove ricerche. Seminario di studi, Roma 2015, pp. 179-217.

Ventura Villanueva A., L'arco di trionfo di Settimio Severo a Roma e le sue iscrizioni con litterae aureae. Una nuova prospettiva, Mitteilungen des Deutschen Archäologischen Institut. Römische Abteilung 120, 2014, pp. 267-312.

Virgili P., Notiziario di scavi e scoperte in Roma e Suburbio 1946-1960, II, Foro di Nerva, Bullettino della Commissione Archeologica Comunale 90, 1985, p. 321. 